\title{
Range-Separated Brueckner Coupled Cluster Doubles Theory
}

\author{
James J. Shepherd, Thomas M. Henderson, and Gustavo E. Scuseria \\ Department of Chemistry and Department of Physics and Astronomy, Rice University, Houston, Texas 77005-1892, USA \\ (Received 23 October 2013; revised manuscript received 21 January 2014; published 3 April 2014; corrected 8 May 2014)
}

\begin{abstract}
We introduce a range-separation approximation to coupled cluster doubles (CCD) theory that successfully overcomes limitations of regular CCD when applied to the uniform electron gas. We combine the short-range ladder channel with the long-range ring channel in the presence of a Bruckner renormalized one-body interaction and obtain ground-state energies with an accuracy of 0.001 a.u./electron across a wide range of density regimes. Our scheme is particularly useful in the low-density and strongly correlated regimes, where regular $\mathrm{CCD}$ has serious drawbacks. Moreover, we cure the infamous overcorrelation of approaches based on ring diagrams (i.e., the particle-hole random phase approximation). Our energies are further shown to have appropriate basis set and thermodynamic limit convergence, and overall this scheme promises energetic properties for realistic periodic and extended systems which existing methods do not possess.
\end{abstract}

DOI: 10.1103/PhysRevLett.112.133002

PACS numbers: 31.15.bw, 71.10.Ca

Introduction.-Physicists have long sought an accurate many-body description of electrons in solids. In this context, the particle-hole random phase approximation (RPA) has a long history in condensed matter physics [1]. Recently, it has seen increased emphasis in the framework of density functional theory (DFT) for electronic structure, where its orbital-based description of electronic correlation has been of great utility [2,3]. One of the most important successes of the RPA lies in the description of dispersion interactions [4], often in range-separated schemes which combine a long-range RPA picture with short-range DFT [5-7]. These range-separated schemes take advantage of the fact that the RPA accurately captures long-range correlations, and tend to mitigate its basis set sensitivity and its known failures for short-range correlations. This failure occurs because RPA predicts too deep a correlation hole and therefore too large a correlation energy $[8,9]$. One popular remedy is the incorporation of second-order screened exchange (SOSEX) effects, as introduced by Freeman [10]. This built on previous work by Gell-Mann and Brueckner, who demonstrated that second-order exchange is required to yield the correct constant term in the high-density expansion for the electron gas [11]. This correction has in recent times been adapted for a wide range of real electronic structure problems $[2,12,13]$.

An alternative approach is motivated by noting that the RPA is an infinite-order summation of the ring diagrams from many-body perturbation theory [11]. From this perspective, the overbinding of the RPA is due to the absence of other diagrams. Amongst these, the ladder diagrams are thought to be the most important [14]. Ladder diagrams have been proposed to yield high-quality short-range correlation functions and have been discussed as an alternative to screened exchange [14]. However, ladder-only theories suffer from the divergences associated with finite-order perturbation theory applied to a Coulombic interaction in metallic solids [15-17].

We here suggest combining the long-range accuracy of RPA with the short-range accuracy of ladder diagrams within a range-separated CCD, based on modern developments relating RPA to CCD [18-21]. We perform a range separation with a Yukawa potential and apply this technique to the electron gas, a model with substantial historical significance $[22,23]$ but nonetheless still receiving prominent attention [24-27]. We show that this method improves both the undercorrelation of CCD and the overcorrelation of RPA, and does so in a manner which accurately captures the high momentum (short distance) approach to the complete basis set limit. We showcase high quality $\left(10^{-3}\right.$ a.u./electron) results at a wide range of densities for finite electron numbers. We also demonstrate this method is suitable for studying the thermodynamic limit.

Theory.-Coupled-cluster doubles (CCD) writes the correlated wave function $|\Psi\rangle$ in an exponential form,

$$
|\Psi\rangle=\mathrm{e}^{T}|0\rangle ; \quad T=\frac{1}{4} t_{i j}^{a b} a_{a}^{\dagger} a_{b}^{\dagger} a_{j} a_{i}
$$

where $|0\rangle$ is a mean-field reference state (typically chosen to be Hartree-Fock), $T$ is an excitation operator and $t$ are amplitudes to be found. Here and throughout, indices $i, j, k$, $l$ label single-particle states occupied in $|0\rangle$ and $a, b, c, d$ label states unoccupied in $|0\rangle$; summation convention is used on dummy indices. Inserting this ansatz into the Schrödinger equation leads to an energy

$$
E=\langle 0|H| 0\rangle+E_{\mathrm{corr}} ; \quad E_{\mathrm{corr}}=\frac{1}{4} t_{i j}^{a b} \bar{v}_{a b}^{i j}
$$

and amplitude equation 


$$
\begin{aligned}
\left(\epsilon_{i}+\epsilon_{j}\right. & \left.-\epsilon_{a}-\epsilon_{b}\right) t_{i j}^{a b}=\bar{v}_{i j}^{a b} \\
& +\bar{v}_{c j}^{k b} t_{i k}^{a c}+\bar{v}_{c i}^{k a} t_{j k}^{b c}+\bar{v}_{c d}^{k l} t_{l j}^{d b} t_{i k}^{a c} \\
& +\frac{1}{2} \bar{v}_{c d}^{a b} t_{i j}^{c d}+\frac{1}{2} \bar{v}_{i j}^{k l} t_{k l}^{a b}+\frac{1}{4} \bar{v}_{c d}^{k l} t_{i j}^{c d} t_{k l}^{a b} \\
& -\bar{v}_{c j}^{k a} t_{i k}^{b c}-\bar{v}_{c i}^{k b} t_{j k}^{a c}-\bar{v}_{c d}^{k l} t_{l j}^{d a} t_{i k}^{b c} \\
& +\frac{1}{2} \bar{v}_{c d}^{k l}\left[t_{l j}^{a b} t_{i k}^{c d}-t_{l i}^{a b} t_{j k}^{c d}+t_{j i}^{d b} t_{k l}^{a c}-t_{i j}^{d a} t_{k l}^{b c}\right],
\end{aligned}
$$

where $\epsilon$ are Hartree-Fock single-particle energies and $\bar{v}_{i j}^{a b}=v_{i j}^{a b}-v_{i j}^{b a}$ is the matrix representation of the electron-electron interaction. Further details can be found in a general review of coupled cluster theory [28].

The terms on the first line of the amplitude equation, Eq. (3) are known as driving terms and arise in a lead-order perturbative expansion of the wave function. The terms on the second line are ring terms which together with the driving terms yield the particle-hole $(p h)$ RPA. Loosely, they treat electronic excitations as bosonic harmonic oscillators. The terms on the third line are ladder terms which together with the driving terms yield the particleparticle $(p p)$ RPA, where they treat pairing excitations as a bosonic oscillator. We note that $p p$-RPA is a less well known type of RPA which is nonetheless familiar to physicists in nuclear structure theory [29].

We call the terms on the fourth line "crossed-ring" terms as they are ringlike but are needed to restore the fermionic antisymmetry of the $T$ amplitudes. The last class of diagrams are what we name the "mosaic" terms which are joint ladderring diagrams that contribute to the Brueckner effective one-body Hamiltonian [30]. Explicitly, we can incorporate these terms into the left hand side of the Eq. (3) as follows:

$$
\eta_{i}=\epsilon_{i}+\frac{1}{2} \bar{v}_{c d}^{i l} t_{i l}^{c d} ; \quad \eta_{a}=\epsilon_{a}-\frac{1}{2} \bar{v}_{a d}^{k l} t_{k l}^{a d} .
$$

Here, we have anticipated this matrix being diagonal in a canonical plane wave basis set due to momentum conservation. The interpretation of this is a renormalization of the single-particle spectrum to account for the effects of correlation. Overall, therefore, coupled-cluster theory combines these channels through a unified set of amplitudes and yields a properly fermionic wave function which incorporates both kinds of RPA fluctuations [20].

Because the $p p$-RPA accurately describes short-range correlations but underestimates long-range correlations, while the conventional $p h$-RPA is instead accurate for long-range correlations and overestimates short-range correlation, a natural approach which combines the benefits of both is to work through a range-separated scheme in which the Coulomb potential is split into a short-range piece to be treated with the ladder diagrams and a long-range piece which is treated with the ring terms. We choose to use a Yukawa separation

$$
\frac{1}{r_{12}}=\frac{e^{-\gamma r_{12}}}{r_{12}}+\frac{1-e^{-\gamma r_{12}}}{r_{12}}
$$

where the parameter $\gamma$ is taken to be the Thomas-Fermi screening parameter. This is an approximation and represents the static limit of the screening from particle-hole RPA and is physically appropriate for metals, though it is well know that this approach is insufficiently screened in semiconductors or insulators [3,31]; it works best when the dielectric properties of the material are homogeneous.

We can now rewrite the CCD amplitude equations replacing the potential with a long-range potential for the ring terms and a short-range potential for the ladder terms and excluding the crossed rings:

$$
\begin{aligned}
\left(\eta_{i}+\eta_{j}-\eta_{a}-\eta_{b}\right) t_{i j}^{a b}= & \bar{v}_{i j}^{a b}+\frac{1}{2}\left(\bar{v}_{\mathrm{SR}}\right)_{c d}^{a b} t_{i j}^{c d}+\frac{1}{2}\left(\bar{v}_{\mathrm{SR}}\right)_{i j}^{k l} t_{k l}^{a b} \\
& +\frac{1}{4}\left(\bar{v}_{\mathrm{SR}}\right)_{c d}^{k l} t_{i j}^{c d} t_{k l}^{a b}+\left(\bar{v}_{\mathrm{LR}}\right)_{c j}^{k b} t_{i k}^{a c} \\
& +\left(\bar{v}_{\mathrm{LR}}\right)_{c i}^{k a} t_{j k}^{b c}+\left(\bar{v}_{\mathrm{LR}}\right)_{c d}^{k l} t_{l j}^{d b} t_{i k}^{a c}
\end{aligned}
$$

From the perspective of the random phase approximation, this is the addition of particle-particle terms with particlehole terms [20]. We remove the crossed rings from these equations because they do not represent an harmonic oscillator problem that can be added into $p p$-RPA or $p h$-RPA [20]. Further technical details are provided as Supplemental Material [32].

Finite basis set electron gas calculations.-The continuum electron gas Hamiltonian is familiar to most, but here it is important to note that we use a basis of plane waves: $\left.\phi_{j} \propto \exp \left(i \mathbf{k}_{j} \cdot \mathbf{r}\right)\right)$.

The momenta, $\mathbf{k}$, associated with these plane waves have a finite spacing specified uniquely by a particle number $(N)$ and a density ( $r_{s}$, the Wigner-Seitz radius). This model is then typically referred to as the finite simulation-cell electron gas. The infinite particle, $N \rightarrow \infty$, limit is referred to as the thermodynamic limit and the energy in this limit is uniquely defined only by the density $r_{s}$. In a frequently used procedure, diffusion Monte Carlo simulations can be made of finite electron numbers and extrapolated to the thermodynamic limit [22,33].

For the methods considered here, we also require an energy cutoff to give a finite number of basis functions $(M)$; even for a finite particle number an infinite basis is required. The extrapolation procedure to reach the complete basis set limit, $M \rightarrow \infty$, has only been recently codified for plane waves $[34,35]$ although these methods have long been in use in quantum chemistry [36].

In summary, the energies we are able to compute have three parameters: $M, N$, and $r_{s}$.

Finite particle, complete basis set limit.-We present results for the 14 electron system at the complete basis set limit in Fig. 1, where we calculate the error of each method shown with respect to quantum Monte Carlo benchmarks [37]. We also make comparison with dRPA + SOSEX, a variant of $p h$-RPA and second-order screened exchange common in condensed matter physics [12,32].

The range separated scheme reported here returns an energy that is consistently within 0.001 a.u. per electron of the exact result, and is of comparable or better accuracy to 


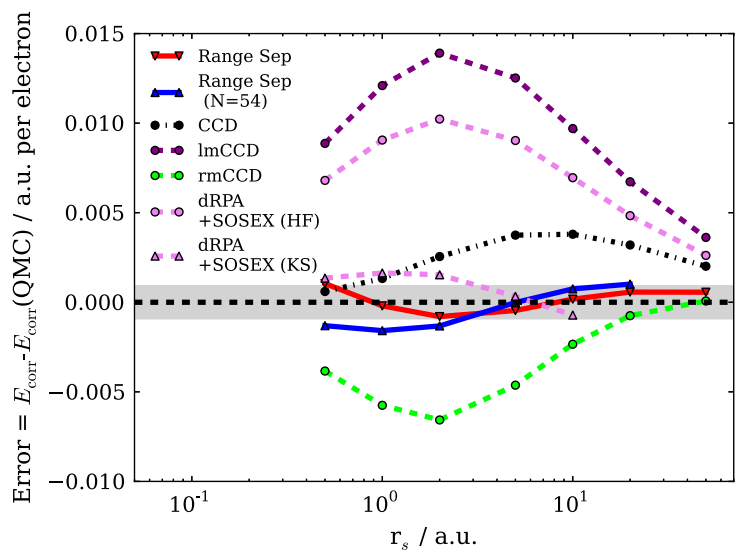

FIG. 1 (color online). Error of the range separated schemes measured with respect to QMC data; the shaded section of the graph represents 0.001 a.u. per electron. (Unless otherwise noted, $N=14$ and $M \rightarrow \infty)$.

dRPA + SOSEX energies and CCD. For more generality we also plot the same curve for the 54 electron system for which only diffusion Monte Carlo results are available [38,39]. Since these only have an accuracy of around 0.001 a.u. per electron in the high density regime [40], the disagreement between the range separation energy and that from QMC calculations is slightly larger.

For further comparison, we provide different CCD calculations where different channels have been excluded. The nomenclature in this paper uses prefixes to denote included channels in a calculation: $r$ for rings, $l$ for ladders, $x$ for crossed rings, and $m$ for mosaics. For example, rmCCD is a CCD calculation performed with the driving, rings and mosaic terms only. The two methods shown, rmCCD (similar to $p h$-RPA) and lmCCD (similar to $p p$-RPA), are known to overcorrelate and undercorrelate, respectively; their combination in range separated CCD performs significantly better.

Basis set convergence.-Although sometimes overlooked, an important aspect of basis set methods is the manner in which the correlation energy is captured as the basis set is expanded. Basis sets which are said to describe correlation consistently describe the physics incrementally and allow for the best extrapolation. For a given Hamiltonian and basis set, this is related to the manner in which a method captures static (strong) versus dynamic (weak) correlation. Exact results from exact diagonalization in a finite basis sets are the best benchmarks to compare behavior between methods.

It has been remarked that dRPA + SOSEX based on a Kohn-Sham reference performs spuriously well for the electron gas [41], in spite of the description of the correlation hole likely being similar to dRPA and hence too deep [8,9]. The overestimation of the correlation energy due to this comes from basis functions with high momenta, and can therefore be seen in the basis set extrapolation curves for large basis set sizes $(M)$ [34]. This is shown in Fig. 2, where a variety of methods are compared. Theories derived from dRPA and second-order perturbation theory (MP2) all have a

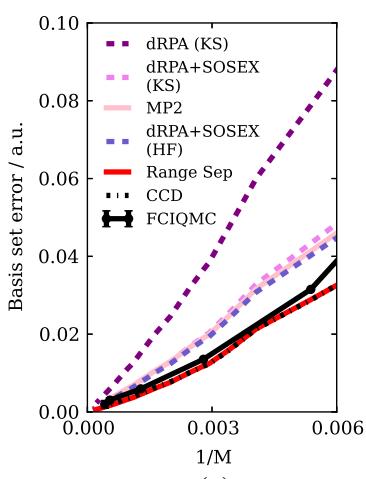

(a)

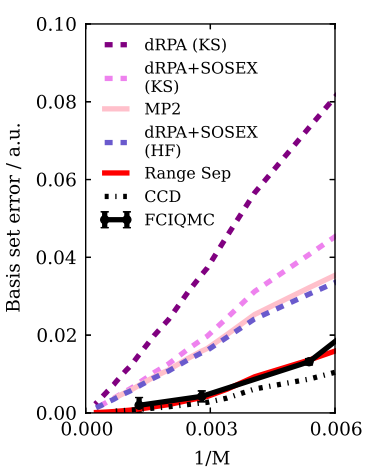

(b)
FIG. 2 (color online). Comparison of approach to the complete basis set limit between different methods at (a) $r_{s}=1.0$ and (b) $r_{s}=5.0$. Error bars on the QMC data are shown at $2 \sigma$, where they better represent $95 \%$ confidence intervals, for emphasis. $(N=14, M \rightarrow \infty$ by extrapolation [42]). Comparison data were drawn from Ref. [34].

behavior that enters the linear $1 / M$ regime too quickly and with too steep a gradient. The quality of the Kohn-Sham dRPA + SOSEX result does not therefore translate to good basis set energies, and therefore can be seen to result from a cancellation of capturing too little correlation energy around the Fermi surface and too much correlation energy at higher momenta.

In contrast, our range-separated scheme retains the much improved behavior of CCD. The benefit of maintaining a single wave function description of the problem, is that we can relate this back to the physical real-space structure of the wave function. The gradient on approach to the complete basis set limit is related to how a method captures the electron correlation holes of the system. Consistency between our proposed method and exact results suggests that this is captured accurately on average between all electrons.

Thermodynamic limit.-In the thermodynamic limit (TDL), finite order perturbation theories can yield divergent energies. One example of this is that the second-order MP2 energy diverges. There has been substantial success in examining these analytically, but with $\mathrm{CC}$ theories this can be very difficult due to the need to solve nonlinear equations. Approaching this limit numerically is also difficult due to slow divergences and the rising cost of simulating additional particles.

We follow a recent method due to Shepherd and Grüneis [43]. In outline, this explored a minimal representation of the area in $k$ space around the Fermi sphere which is the origin of the low-momentum excitations that cause the divergence. This amounts to performing a numerical quadrature of a small area around the Fermi sphere (typically of radius $\sqrt{2}$ larger) by more and more finely spaced grids in such a way that the divergence can be seen by constant and predictable growth of the energy. By a judicious choice of basis set sizes for each system size, computational cost can be controlled. Although only a small fraction of the total energy is represented in this region, it was shown to be enough to demonstrate the divergence in the energy. 


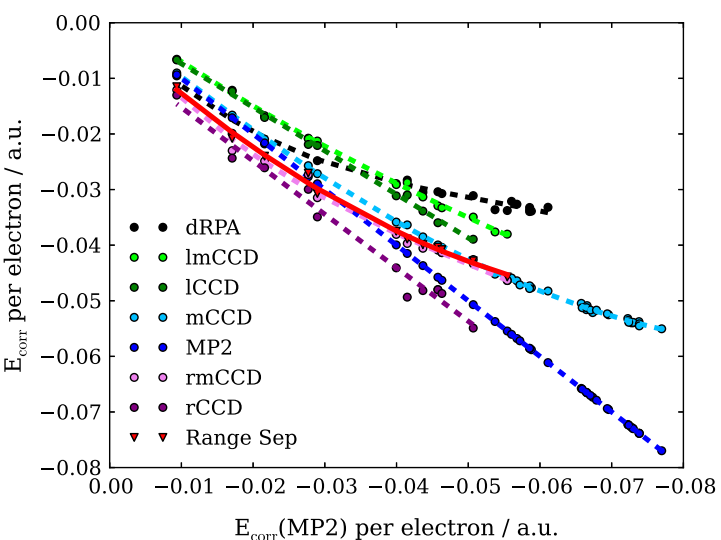

(a)

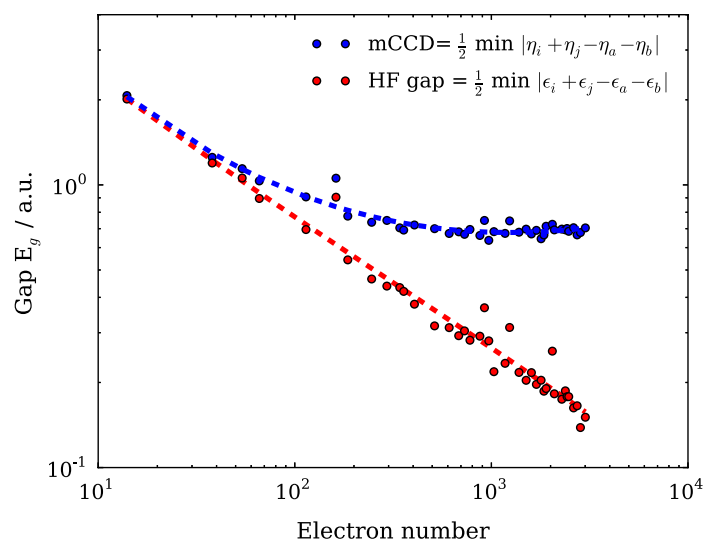

(b)

FIG. 3 (color online). Behavior on approach to the TDL. Each point on this graph represents a single $N$ and $M$ and values vary between $N=14-3006$ with $M=38-8338$; the gas density was $r_{s}=1.0$ a.u. In (a), we compare energies. Divergent methods appear as a straight line, apparent convergences as a curve. The lines only represent fits after the method is determined to converge or diverge, and are only intended as a guide to the eye. In (b), we compare the orbital energy gap HF and the same gap in mCCD; the latter remains positive and finite in the TDL (see text). Comparison data are explained in more depth in Ref. [44].

Figure 3(a) plots such an analysis for various methods described in this paper. MP2 is well understood to have a divergent energy and can be used therefore as a divergence benchmark. We plot MP2 energies against energies obtained with different theories. A correlation with MP2 suggests that the method in question diverges at the same speed as MP2. Since all higher order divergences are quicker, the appearance of a deviation from correlation with MP2 implies a convergent energy. This is exemplified by dRPA. Our companion study discusses these limitations more thoroughly [44].

As expected, the dRPA does not have a linear correlation with MP2, nor does CCD which would overlay the dRPA line on this scale. The ladder-only diagrams (ICCD) diverge, which is also consistent with a variety of previous comments and discussions in the literature (e.g., [15]). More surprisingly, the same appears true for rCCD which is subtly different from dRPA [18]. In general, however, methods can be made to converge by the inclusion of mosaic diagrams and changing the reference for the calculation to the Brueckner Hamiltonian. This can be seen most dramatically by comparing pairs of lines with and without mosaics: rCCD with rmCCD; ICCD with $\operatorname{lmCCD}$; and MP2 with mCCD. This test also shows that the range-separated scheme converges, as desired.

Finite electron gases have gapped orbital energy spectra due to the finite spacing of $k$ states. The orbital energy gap calculated from Hartree-Fock theory, i.e.,

$$
E_{g}=\frac{1}{2} \min \left|\epsilon_{i}+\epsilon_{j}-\epsilon_{a}-\epsilon_{b}\right|,
$$

closes in the TDL, which can be seen by examining the gap for a series of system sizes as shown in Fig. 3(b). The closure of this orbital energy gap is one of the reasons that perturbation theories diverge for metals, since the smallest energy denominator is just twice the smallest orbital energy gap. In contrast, adding mosaic terms yields a modified single-particle spectrum which remains gapped even in the TDL, which suppresses the divergences, though we must point out that this nonzero orbital energy gap does not imply that the many-particle wave function is insulating. More discussion of this is included in the Supplemental Material [32].

Conclusion.-We have made an approximation of the CCD equations using ideas inspired from range separation in density functional theory and applied it to finite electron gas systems. We find a combination of terms which couple together $p p$-RPA in the short range and $p h$-RPA in the long range and in a manner that is superior to both methods.

This allows us to propose an approximation with the same computational cost scaling as CCD with several desirable properties: (a) improved accuracy over CCD, especially in the low-density regime; (b) improved accuracy over various forms of dRPA + SOSEX, especially in calculating energies from high-momentum basis functions; (c) retention of a finite energy per particle in the thermodynamic limit. This method performs well over a wide range of density regimes. The formulation of this method in terms of the coupled cluster wave function ansatz should allow for further investigation of solid state properties using tools and methods built upon this framework. Therefore, we hope that the method presented here will be transferable to real periodic and extended systems, where there is a growing interest in treating problems in solid state materials science with wave function and many-body methods.

The authors thank Andreas Savin and Andreas Grüneis for helpful discussions and the authors of Ref. [45] for early access to their data. One of us (J. J. S.) would like to thank the Royal Commission for the Exhibition of 1851 for a Research Fellowship. This work was supported by the National Science Foundation (CHE-1102601), the Welch Foundation (C-0036), and DOE-CMCSN (DESC0006650). 
*jjs6@rice.edu

[1] D. Bohm and D. Pines, Phys. Rev. 82, 625 (1951); D. Pines and D. Bohm, Phys. Rev. 85, 338 (1952); D. Bohm and D. Pines, Phys. Rev. 92, 609 (1953).

[2] J. Harl and G. Kresse, Phys. Rev. Lett. 103, 056401 (2009).

[3] T. Leininger, H. Stoll, H.-J. Werner, and A. Savin, Chem. Phys. Lett. 275, 151 (1997); E. Goll, H.-J. Werner, and H. Stoll, Phys. Chem. Chem. Phys. 7, 3917 (2005); E. Goll, H.-J. Werner, H. Stoll, T. Leininger, P. Gori-Giorgi, and A. Savin, Chem. Phys. 329, 276 (2006); S. Lebègue, J. Harl, T. Gould, J. G. Ángyán, G. Kresse, and J. F. Dobson, Phys. Rev. Lett. 105, 196401 (2010).

[4] J. F. Dobson, A. White, and A. Rubio, Phys. Rev. Lett. 96, 073201 (2006).

[5] J. Toulouse, I. C. Gerber, G. Jansen, A. Savin, and J. G. Ángyán, Phys. Rev. Lett. 102, 096404 (2009).

[6] B. G. Janesko, T. M. Henderson, and G. E. Scuseria, J. Chem. Phys. 130, 081105 (2009).

[7] F. Bruneval, Phys. Rev. Lett. 108, 256403 (2012).

[8] K. S. Singwi, M. P. Tosi, R. H. Land, and A. Sjölander, Phys. Rev. 176, 589 (1968).

[9] S. Kurth and J. P. Perdew, Phys. Rev. B 59, 10461 (1999).

[10] D. L. Freeman, Phys. Rev. B 15, 5512 (1977).

[11] M. Gell-Mann and K. A. Brueckner, Phys. Rev. 106, 364 (1957).

[12] H. Eshuis, J. E. Bates, and F. Furche, Theor. Chem. Acc. 131, 1 (2012).

[13] J. Paier, X. Ren, P. Rinke, G. E. Scuseria, A. Grüneis, G. Kresse, and M. Scheffler, New J. Phys. 14, 043002 (2012).

[14] R. F. Bishop and K. H. Lührmann, Phys. Rev. B 17, 3757 (1978); 26, 5523 (1982); R. F. Bishop, Theor. Chim. Acta 80, 95 (1991).

[15] D. L. Freeman, J. Phys. C 16, 711 (1983).

[16] J. Cioslowski and P. Ziesche, Phys. Rev. B 71, 125105 (2005).

[17] N. D. Drummond and R. J. Needs, Phys. Rev. B 79, 085414 (2009).

[18] G. E. Scuseria, T. M. Henderson, and D. C. Sorensen, J. Chem. Phys. 129, 231101 (2008).

[19] D. Peng, S. N. Steinmann, H. van Aggelen, and W. Yang, J. Chem. Phys. 139, 104112 (2013).

[20] G. E. Scuseria, T. M. Henderson, and I. W. Bulik, J. Chem. Phys. 139, 104113 (2013).

[21] H. van Aggelen, Y. Yang, and W. Yang, Phys. Rev. A 88, 030501 (2013).

[22] D. M. Ceperley and B. J. Alder, Phys. Rev. Lett. 45, 566 (1980).

[23] J. P. Perdew and A. Zunger, Phys. Rev. B 23, 5048 (1981).

[24] S. Huotari, J. A. Soininen, T. Pylkkänen, K. Hämäläinen, A. Issolah, A. Titov, J. McMinis, J. Kim, K. Esler, D. M. Ceperley, M. Holzmann, and V. Olevano, Phys. Rev. Lett. 105, 086403 (2010).

[25] N. D. Drummond, P. López Ríos, R. J. Needs, and C. J. Pickard, Phys. Rev. Lett. 107, 207402 (2011).

[26] M. Holzmann, B. Bernu, C. Pierleoni, J. McMinis, D. M. Ceperley, V. Olevano, and L. Delle Site, Phys. Rev. Lett. 107, 110402 (2011).

[27] L. Baguet, F. Delyon, B. Bernu, and M. Holzmann, Phys. Rev. Lett. 111, 166402 (2013).
[28] R. J. Bartlett and M. Musiał, Rev. Mod. Phys. 79, 291 (2007).

[29] P. Ring and P. Schuck, The Nuclear Many-Body Problem (Springer-Verlag, Berlin, 1980).

[30] G. E. Scuseria, Int. J. Quantum Chem. 55, 165 (1995).

[31] T. Shimazaki and Y. Asai, Chem. Phys. Lett. 466, 91 (2008).

[32] See Supplemental Material http://link.aps.org/supplemental/ 10.1103/PhysRevLett.112.133002 for further discussions, which includes Refs. [20,22,30,34,35,39,40,42,43,45-54].

[33] N. D. Drummond, R. J. Needs, A. Sorouri, and W. M. C. Foulkes, Phys. Rev. B 78, 125106 (2008).

[34] J. J. Shepherd, A. Grüneis, G. H. Booth, G. Kresse, and A. Alavi, Phys. Rev. B 86, 035111 (2012).

[35] A. Grüneis, J. J. Shepherd, A. Alavi, D. P. Tew, and G. H. Booth, J. Chem. Phys. 139, 084112 (2013).

[36] C. Hättig, W. Klopper, A. Köhn, and D. P. Tew, Chem. Rev. 112, 4 (2012).

[37] For $N=14$, the benchmarks here come from full configuration interaction quantum Monte Carlo [50] for the high-tometallic density regime and diffusion Monte Carlo for the remainder [48,51]. These results are effectively exact following extensive development and benchmarking [34,40,42,43].

[38] Y. Kwon, D. M. Ceperley, and R. M. Martin, Phys. Rev. B 58, 6800 (1998).

[39] P. López Ríos, A. Ma, N. D. Drummond, M. D. Towler, and R. J. Needs, Phys. Rev. E 74, 066701 (2006).

[40] J. J. Shepherd, G. Booth, A. Grüneis, and A. Alavi, Phys. Rev. B 85, 081103 (2012).

[41] A. Grüneis, M. Marsman, J. Harl, L. Schimka, and G. Kresse, J. Chem. Phys. 131, 154115 (2009).

[42] J. J. Shepherd, G. H. Booth, and A. Alavi, J. Chem. Phys. 136, 244101 (2012).

[43] J. J. Shepherd and A. Grüneis, Phys. Rev. Lett. 110, 226401 (2013).

[44] J. J. Shepherd, T. M. Henderson, and G. E. Scuseria, J. Chem. Phys. 140, 124102 (2014).

[45] J. J. Shepherd, P. L. Ríos, N. D. Drummond, R. J. Needs, and A. Alavi (to be published); J. J Shepherd, P. L. Ríos, R. J. Needs, R. J. Needs, J. A.-F. Mohr, G. H. Booth, A. Grüneis, G. Kresse, A. Alavi, APS Meeting Abstracts 1, 24003 (2013).

[46] R. J. Bartlett, Chem. Phys. Lett. 484, 1 (2009).

[47] L. M. Fraser, W. M. C. Foulkes, G. Rajagopal, R. J. Needs, S. D. Kenny, and A. J. Williamson, Phys. Rev. B 53, 1814 (1996).

[48] R. J. Needs, M. D. Towler, N. D. Drummond, and P. López Ríos, J. Phys. Condens. Matter 22, 023201 (2010).

[49] M. Holzmann, D. M. Ceperley, C. Pierleoni, and K. Esler, Phys. Rev. E 68, 046707 (2003).

[50] G. H. Booth, A. Grüneis, G. Kresse, and A. Alavi, Nature (London) 493, 365 (2013).

[51] P. López Ríos, Pers. Comm. (2013).

[52] R. D. Mattuck, A Guide to Feynman Diagrams in the Many-Body Problem, Dover Books on Physics and Chemistry (Dover, New York, 1992), 2nd ed..

[53] A. Grüneis, M. Marsman, and G. Kresse, J. Chem. Phys. 133, 074107 (2010).

[54] R. M. Martin, Electronic Structure: Basic Theory and Practical Methods (Cambridge University Press, Cambridge, England, 2004). 